\title{
Simulation study of traffic accidents in bidirectional traffic models
}

\author{
Najem Moussa * \\ Département de Mathématique et Informatique, \\ Faculté des Sciences, B.P. 20 - 24000 - El Jadida, Morocco
}

May 28, 2018

\begin{abstract}
Conditions for the occurrence of bidirectional collisions are developed based on the Simon-Gutowitz bidirectional traffic model. Three types of dangerous situations can occur in this model. We analyze those corresponding to head-on collision, rear-end collision and lanechanging collision. Using Monte Carlo simulations, we compute the probability of the occurrence of these collisions for different values of the oncoming cars density. It is found that the risk of collisions is important when the density of cars in one lane is small and that of the other lane is high enough. The influence of different proportions of heavy vehicles is also studied. We found that heavy vehicles cause an important reduction of traffic flow on the home lane and provoke an increase of the risk of car accident.
\end{abstract}

Pacs numbers: 89.40.+k; 02.60.Cb; 45.70.Vn; 89.75.Fb

Keywords: Cellular automata; bidirectional traffic; heavy vehicle; head-on collision, rear-end collision; Lane-changing collision.

\section{Introduction}

In the past few years, the investigation of traffic flow using cellular automata (CA) has become quite popular [1, 2]. The most popular CA model for

*e-mail: najemmoussa@yahoo.fr 
traffic flow on one-lane roadway is the Nagel-Schreckenberg (NS) model [3]. Despite its simplicity, the model is capable of capturing some essential features observed in realistic traffic like density waves or spontaneous formation of traffic jams. After that, various generalizations and extensions of this onelane model have been considered and different other congested traffic states may occur such as synchronous phase, wide moving jams and stop-and-go phase [4, 5].

The NS model rule set was extended to a multi-lane model and has been applied to the investigation of traffic flow in highways [6-9]. A CA model of bidirectional vehicular traffic by Simon and Gutowitz (SG) extends the case to vehicles moving in opposite directions on a two-lane road where passing is allowed [10]. When a driver encounters a slower forward moving vehicle, a pass will be attempted. To do this, driver checks the density of vehicles in front that have to be passed, i.e. the local density. If this density is low enough the pass will be performed on condition of checking the safety criteria on the oncoming lane. If the density of cars in one lane is small and that of the other lane is high enough then wide jams occur in both lanes. The occurrence of these wide jams are due principally to the fact that almost all passing cars get stuck with oncoming cars before returning to their home lanes. Recently, the SG model for bidirectional traffic flow is revisited where some rules are changed in order to prevent the occurrence of wide moving jams [11].

In modern society, traffic accidents and congestion continue to plague the transportation managers. Accident analysis and prevention is one of the most important aspects of interest in traffic management because it is associated with human life. Recently, cellular automata models have been used to investigate the probability of the occurrence of car accidents. Boccara et al. 12] have been the first authors to propose conditions for car accidents to occur in the deterministic NS model. The first condition is that the number of empty cells in front of the car (gap) is smaller than the speed limit, the second condition is that the car ahead is moving, and the last condition is that the moving car ahead is suddenly stopped at the next time step. Using these conditions, analytic results of the probability of car accidents are obtained in some special cases of the NS model with periodic boundary conditions [13, 14]. General numerical results concerning the probability of car accidents are reported for the nondeterministic NS model [15-17]. The probability for an accident to occur is found to be proportional to the product of the fraction of stopped cars and the traffic flow [18]. Conditions for 
the occurrence of car accidents in the NS model are developed based on the driver reaction time [19]. The probability for car accidents to occur has been extensively investigated in the velocity effect model [20], two-lane CA model [21], NS model with open boundaries [22], NS model with speed limit zone [23], NS model with velocity-dependent randomization [24] and NS model with quenched randomness [25, 26]

The purpose of the present paper is precisely the investigation of the probability of collisions in bidirectional traffic models and to analyze the effect of heavy vehicles on the traffic flow and on the probability of car accidents. The paper is organized as follows. In Sec. 2, we describe the model for bidirectional traffic. Here, we consider the SG bidirectional model revisited [11]. In Sec. 3, we introduce conditions for the occurrence of car accidents. In Sec. 4, we present our simulation results and discussions. Finally, we conclude with a summary in Sec. 5 .

\section{Model}

The SG model is a probabilistic CA which consists of vehicles moving on two opposite lanes of $L$ cells with periodic boundary conditions. Each cell is either empty, or occupied by just one vehicle. In our model, there exist two types of lanes: lane ${ }^{[+]}$and lane $e^{[-]}$. On lane $e^{[+]}$, two kinds of vehicles move from left to right: cars and heavy vehicles. We suppose that passing is forbidden for heavy vehicles and only the cars can change lanes. In the model, we suppose that no heavy vehicles exist on lane ${ }^{[-]}$and only cars move from right to left. We denote by vehicle $e^{[+]}$the vehicle moving on the lane ${ }^{[+]}$ with positive direction and vehicle ${ }^{[-]}$the vehicle moving on the lane ${ }^{[-]}$with negative direction (see figure 1). We denote by $x$ and $v$ the position and the speed of a vehicle at time $t$ respectively. To distinguish between cars and heavy vehicles, we suppose that the maximal speed of the heavy vehicles is lower to the one of the cars. Hence, we set $v_{\max }=3$ for heavy vehicles and $v_{\max }=5$ for cars. To distinguish between different interacting vehicles, several gaps and speeds are introduced. gap $_{\text {same }}\left(\right.$ gap $\left._{\text {opp }}\right)$ : the number of unoccupied sites in front of a vehicle on the same (opposite) lane. gap behind: the number of unoccupied sites behind the vehicle, on the opposite lane. $v_{\text {same }}$ $\left(v_{\text {opp }}\right)$ : the speed of the vehicle ahead on the same (opposite) lane. On the aim of making more compact the rules of the bidirectional model, several logical functions are introduced. $\mathbf{H}$ : true if the vehicle is on its home lane. $\mathbf{T}$ : true 
if the vehicle is a heavy vehicle. oncoming: true if vehicle ${ }^{[+]}$and vehicle $\mathrm{e}^{[-]}$ are face-to-face on the same lane. Space1: true if $\left(\right.$ gap $\left._{\text {same }}<l_{\text {pass }}\right)$ AND $\left(\right.$ gap $\left._{\text {opp }}>l_{\text {security }}\right)$ AND $\left(\right.$ gap $\left._{\text {behind }}>l_{\text {back }}\right)$. Space2: true if gap behind $>l_{\text {back }}$. The parameters $l_{\text {pass }}, l_{\text {back }}$ and $l_{\text {security }}$ are defined by the following. $l_{\text {pass }}$ : if gap $_{\text {same }}<l_{\text {pass }}$ AND $\mathbf{H}$ then a pass may be attempted. $l_{\text {back }}$ : the distance a driver looks back for obstacles on the passing lane. $l_{\text {security }}$ : if gap same $_{<}<$ $l_{\text {security }}$ AND not $(\mathbf{H})$ then the vehicle returns immediately to its home lane. $D_{L}$ : local density: the fraction of the $l_{\text {density }}=2 v_{\max }+1$ sites in front of the given vehicle which are occupied; $D_{\text {limit }}=1 / l_{\text {density }}$ : the maximum local density for a safe pass.

At each discrete time-step $t \rightarrow t+1$ the system update is performed in parallel for all cars according to the following subrules :

\section{i. Lane changing rules:}

1. IF $\left(\operatorname{not}(\mathbf{T})\right.$ AND $\mathbf{H}$ AND Space1 AND $\left(D_{L} \leq D_{\text {limit }}\right)$ AND $($ rand $<$ $\left.\left.p_{\text {change }}\right)\right)$ THEN change lane

2. IF $\left(\operatorname{not}(\mathbf{T})\right.$ AND not $(\mathbf{H})$ AND $\left(\left(\right.\right.$ gap $\left._{\text {same }}<l_{\text {security }}\right)$ OR Space2 $\left.)\right)$ THEN change lane.

The first condition concerns cars on their home lane that want to change lane. When a driver encounters a slower forward moving vehicle, a pass is attempted. However, the pass will only be initiated if there is room far enough ahead on the passing lane, and the number of vehicles in front of the car it would like to pass is small. Passing occurs randomly, even if all these conditions are met; the probability of changing lanes is denoted $p_{\text {change }}$. The second condition concerns cars in the midst of passing. They return to their home lane if forced to by an oncoming vehicle, or if there is space enough on the home lane that they can return without braking.

ii. Forward moving rules:

1. IF $\left(v \neq v_{\max }\right)$ THEN $v=v+1$

2. $\operatorname{IF}\left((\right.$ oncoming $)$ AND $\left(\right.$ gap $\left.\left._{\text {same }} \leq\left(2 v_{\max }-1\right)\right)\right)$ THEN $v=g a p_{\text {same }} / 2$

3. IF ( (not(oncoming) $)$ AND $\left(v>\right.$ gap $\left.\left._{\text {same }}\right)\right)$ THEN $v=$ gap $_{\text {same }}$

4. IF ( $\mathbf{H ~ A N D ~}(v \geq 1)$ AND (rand $\left.<p_{\text {decel }}\right)$ AND not(oncoming) $)$ THEN $v=v-1$

5. IF (H AND (oncoming) AND $(v \geq 1)$ ) THEN $v=v-1$.

If the vehicle is a vehicle ${ }^{[+]}$then the vehicle moved forward according to: $x \leftarrow x+v$. But, if the vehicle is a vehicle ${ }^{[-]}$then the vehicle moved backward according to $x \leftarrow x-v$.

The rule (1) reflects the tendency of drivers to drive as fast as possible without exceeding the maximum speed limit. Rule (2) rapidly decelerates the vehicle 
if there is an oncoming vehicle too close. Rule (3) is intended to avoid collision between the vehicles of the same type. Rule (4) randomly decelerates the vehicle if it is on its home lane; if it is passing, it never decelerates randomly. Finally, rule (5) breaks the symmetry between the lanes, and thus prevents the emergence of a super jam, i.e., a jam which may occur when each of an adjacent pair of vehicle ${ }^{[+]}$and vehicle ${ }^{[-]}$, one on each lane, tries to pass simultaneously.

\section{Conditions for the occurrence of car acci- dents}

In order to make effective plans or strategies of comprehensive traffic safety, it is important to evaluate objectively the risk of traffic accidents. However, the empirical data of car accidents cannot be established from real life traffic since the traffic accident itself is a kind of rare event. In reality, no one can make real crash tests with living drivers. Thus, theoretical investigations using statistical physics are highly desirable to evaluate the effects of traffic safety in terms of number of traffic accident. Recently, cellular automata models have been extended to investigate the probability of car accidents in unidirectional traffic roads [12-26]. In this paper, we shall investigate the probability of car accidents in bidirectional traffic flow models. We distinguish three types of car accidents: rear-end collision, lane-changing collision and head-on collision.

i. Rear-end collision is between two vehicles evolving in the same lane and with the same direction. Based on the delayed reaction time of the successor vehicle and the unexpected abrupt deceleration of the predecessor vehicle, the conditions for the occurrence of rear-end collision are expressed [19]. When the vehicle ahead with speed $v_{k+1}^{[+]}(t)$ do an abrupt deceleration, its

velocity will be reduced to $v_{k+1}^{[+]}(t+1)$ at time $t+1$. Thus, if the covered distance during the delayed reaction time $\tau$ of the successor vehicle is enough to reach the next time position of the vehicle ahead, rear-end collision happens most likely on the road. Hence, the conditions for the occurrence of rear-end collision with respect to abrupt deceleration of the vehicle ahead 
are as follows:

$$
\begin{array}{r}
\tau v_{k}^{[+]}(t)>\operatorname{gap}_{\text {same }}^{k}(t)+v_{k+1}^{[+]}(t+1) \\
v_{k+1}^{[+]}(t)-v_{k+1}^{[+]}(t+1) \geq v_{d}
\end{array}
$$

where $\operatorname{gap}_{\text {same }}^{k}(t)$ is the number of unoccupied sites in front of the vehicle $(\mathrm{k})$ on the same lane at time t. The parameter $v_{d}$ is the deceleration limit beyond which a risk of the occurrence of rear-end collision exists.

ii. Lane-changing collision is between a vehicle ${ }^{[+]}$on lane ${ }^{[-]}$that want to return to its home lane and its following vehicle ${ }^{[+]}$on lane ${ }^{[+]}$. An unexpected lane changing of a vehicle ${ }^{[+]}$may lead to a collision on lane ${ }^{[+]}$, if the following vehicle $^{[+]}$cannot decelerate enough [21]. In terms of delayed reaction time $\tau$ of the following vehicle, we can express the conditions for the occurrence of lane-changing collision as:

$$
\begin{gathered}
\operatorname{vehicle}_{(k+1)}^{[+]} \text {changes lane at time } t \\
\tau v_{k}^{[+]}(t)>g a p_{\text {same }}^{k}(t)+v_{k+1}^{[+]}(t+1)
\end{gathered}
$$

If the distance covered during the delayed reaction time $\tau$ of the following vehicle $^{[+]}(\mathrm{k})$ on lane ${ }^{[+]}$is enough to reach the next time position of the vehicle $^{[+]}$ahead that changed lanes $(\mathrm{k}+1)$, lane-changing crash happens most likely on lane ${ }^{[+]}$.

iii. Head-on collision is between a vehicle ${ }^{[+]}$and a vehicle ${ }^{[-]}$that evolve on the same lane and opposites directions. This type of collision leads usually to fatal injuries. The conditions for the occurrence of head-on collision are:

$$
\begin{aligned}
& \text { vehicle }^{[+]} \text {and vehicle }{ }^{[-]} \text {are face to face } \\
& \qquad \tau v_{k}^{[+]}(t)>\operatorname{gap}_{\text {same }}^{k}(t)-v_{k+1}^{[-]}(t+1)
\end{aligned}
$$

If the distance covered during the delayed reaction time $\tau$ of the vehicle ${ }^{[+]}$ $(\mathrm{k})$ is enough to reach the next time position of the vehicle ${ }^{[-]}(\mathrm{k}+1)$ ahead, which moves in the opposite direction, fatal car accident happens most likely.

\section{Numerical results}

We simulate the bidirectional traffic model described above on a lattice of $L$ sites with closed boundary conditions. The density $\rho^{[+]}\left(\right.$resp. $\left.\rho^{[-]}\right)$is 
defined as $\rho^{[+]}=N^{[+]} / L$ (resp. $\left.\rho^{[-]}=N^{[-]} / L\right)$, where $N^{[+]}\left(\right.$resp. $\left.N^{[-]}\right)$is the number of vehicles ${ }^{[+]}$(resp. vehicles ${ }^{[-]}$). We denote by $N_{H V}^{[+]}$the number of heavy vehicles in the circuit. In this article, without losing generality, the model parameters are assumed to be as follows: $l_{\text {pass }}=v, l_{\text {back }}=v_{\text {max }}$, $l_{\text {security }}=2 v_{\max }+1, p_{\text {change }}=0.5$, and $p_{\text {decel }}=0.3$. The maximal velocity of the cars is $v_{\max }=5$ and that of the heavy vehicles is $v_{\max }=3$. The system size is given by $L=2000$. The delayed reaction time is assumed to be $\tau=1 \mathrm{~s}$ and the deceleration limit is $v_{d}=2$.

\subsection{Traffic flow and lane-changing frequency}

Clearly, the traffic flow of vehicles ${ }^{[+]}$, the lane-changing frequency of $\operatorname{cars}^{[+]}$ and the occurrence of dangerous situations, all should depend on the traffic density on the oncoming lane. As in reference [11], we shall analyze the case of two different oncoming lane densities. So, we choose $\rho^{[-]}=0.05$ and $\rho^{[-]}=0.30$, representing densities below and above the critical density in the NS model, respectively. As in unidirectional traffic models, when $\rho^{[+]}$is low, the presence of heavy vehicles in the circuit leads to the formation of platoon of cars behind the heavy vehicles. Yet, the presence of only one heavy vehicle can lead to a drastic reduction of the average flow in the circuit. When $\rho^{[-]}$ is small enough, the flow on the home lane is reduced for low values of $\rho^{[+]}$. But, the flow on the oncoming lane increases with increasing the fraction of heavy vehicles (Fig. 2a). Indeed, the presence of heavy vehicles in the home lane encourages the pass of cars $^{[+]}$on the oncoming lane. If $\rho^{[-]}$is relatively high, passing of $\operatorname{cars}^{[+]}$become rare and the flow on the oncoming lane will be very small (Fig. 2b). Clearly, the heavy vehicles dominate the average flow on the home lane as like as in one-lane systems.

In figures $3 \mathrm{a}$ and $3 \mathrm{~b}$, we show the lane-changing frequency of $\operatorname{cars}^{[+]}$, as a function of $\rho^{[+]}$, for different values of $\rho^{[-]}$. When $\rho^{[-]}$is low, and in the absence of heavy vehicles, the lane-changing rate of $\mathrm{cars}^{[+]}$is maximal at density $\rho^{[+]}$around the critical density separating the free-flow and congested regimes in the NS model (Fig. 3a). If one heavy vehicle is present in the circuit, almost all the $\operatorname{cars}^{[+]}$are organized into platoon behind the heavy vehicle. Lane-changing rate of the cars $^{[+]}$will be weakened, compared to the situation where no heavy vehicle is present, because of the reduction of their speeds and the higher value of their local densities. Additional heavy vehicles may divide the platoon into small ones and then decreases the local densities. This may increase the lane-changing rate of $\operatorname{cars}^{[+]}$and then their 
flow will increase on the oncoming lane. In the case where $\rho^{[-]}$is high enough, passing of cars $^{[+]}$become rare and the lane-changing frequency will be very small (Fig. 3b). Moreover, we find that higher values of the lane-changing frequency occur at very low values of $\rho^{[+]}$. Since free available spaces may exist on lane ${ }^{[+]}$, cars $^{[-]}$can pass other cars $^{[-]}$and then impede traffic on lane $^{[+]}$. In this situation, lane-changing maneuvers can be performed by the fast cars $^{[+]}$in order to overtake the slow ones. However, the presence of heavy vehicles decreases the velocities of the overall cars $^{[+]}$; leading therefore to a decrease of the lane-changing rate.

\subsection{Rear-end collision}

A rear-end collision is a traffic accident where a vehicle hits the rear of the vehicle in front of it. Rear-end collision is mainly caused by inattentive driving behavior and not keeping proper distance from the preceding vehicle. The resulting injuries tended to be of low severity. In our model, the dangerous situation corresponding to the rear-end collision exists when the conditions 1 and 2 occur simultaneously. The probability of rear-end collision (PRC) as a function of $\rho^{[+]}$, for different values of $\rho^{[-]}$, is illustrated in figures $4 \mathrm{a}$ and 4b. First, we analyze the situation where $\rho^{[-]}$is low enough. When no heavy vehicle is present in the home lane, the PRC is as like as in one-lane traffic model [19]. That is, there exists a critical density $\rho_{c}^{[+]}$situated in the lowdensity region, below which no rear-end collision can occur. With increasing $\rho^{[+]}$, PRC increases, reaches a maximum, then decreases and vanishes when $\rho^{[+]}$exceeds some high value $\rho_{h}^{[+]}$. Nevertheless, the presence of only one heavy vehicle provokes an important increase of the risk of rear-end collision over a wide range of the home lane density (fig. 4a). Indeed, the presence of heavy vehicles provokes the creation of traffic jams and platoons of cars in the home lane. This will promotes the lane-changing of cars $^{[+]}$in order to overtake the leading heavy vehicles (see fig. 3a). In this situation, rear-end collisions occur more likely, because a vehicle suddenly decelerates due to an unexpected deceleration of the jammed vehicles ahead.

In figure $4 b$, we show simulation results for the PRC in the case where $\rho^{[-]}$ is high enough. First, we analyze the situation when the heavy vehicles are absent in the circuit. So, we found that the risk of rear-end collision exists even at very low home-lane density. If $\rho^{[+]}$is low, free available spaces exist on lane ${ }^{[+]}$and some $\operatorname{cars}^{[-]}$pass other $\operatorname{cars}^{[-]}$and then impede traffic on lane ${ }^{[+]}$. This causes an important deceleration manoeuvres of vehicles ${ }^{[+]}$. 
Thus, in contrast to one-lane unidirectional models, an important value of the risk of rear-end collision will be observed, even at very low values of $\rho^{[+]}$. When heavy vehicles are present, the fraction of both passing cars $^{[+]}$and passing cars $^{[-]}$will increase; leading therefore to the creation of more jams in both lanes. This may enhance the probability of the occurrence of rear-end dangerous situations.

\subsection{Lane-changing collision}

In addition to rear-end collisions, personal injuries often times result when one vehicle makes an improper lane change and makes contact with the vehicle in the adjacent lane. The lane-changing collision produces injuries and damage which are often severe. In our model, lane-changing collision occurs more likely when a passing car $^{[+]}$performs an unexpected return to the home lane. The dangerous situation corresponding to the lane-changing collision exists when the conditions 3 and 4 occur simultaneously. Thus, it is obvious that the probability of lane-changing collision (PLC) depends straightforwardly on the frequency of lane-changing of $\operatorname{cars}^{[+]}$. The PLC as a function of $\rho^{[+]}$, for different values of $\rho^{[-]}$, is illustrated in figures $5 \mathrm{a}$ and $5 \mathrm{~b}$. In figure $5 \mathrm{a}$, we show simulation results for the PLC in the case where

$\rho^{[-]}$is low enough. So, we observe that, at low values of $\rho^{[+]}$, the risk of lanechanging collision is very low in the case where heavy vehicles are absent; but it is very high when at least one heavy vehicle is present in the circuit. At very low densities, we found that increasing the number of heavy vehicles has no effect on the rate of lane-changing accidents. At relatively high density of vehicles $^{[+]}$, the influence of the number of heavy vehicles is noticeable. That is, we see that the PLC increases considerably with increasing the fraction of heavy vehicles. At higher densities, the influence of heavy vehicles becomes weak and disappears completely beyond certain value of $\rho^{[+]}$.

In figure $5 \mathrm{~b}$, we show simulation results for the PLC in the case where $\rho^{[-]}$ is high enough. We first observe that the risk of lane-changing collision is very low compared to the case where $\rho^{[-]}$is low enough. We find also that the PLC is almost nonexistent when there is no heavy vehicles in the circuit. The PLC increases with increasing the number of heavy vehicles; and more importantly near the critical density. 


\subsection{Head-on collision}

A head-on collision typically occurs when a vehicle executes a passing manoeuvre and crashes into an oncoming vehicle. These type of collisions are often fatal. The occurrence of both conditions 5 and 6 can be a potentially dangerous situation of head-on collision. The probability of head-on collision $(\mathrm{PHC})$ as a function of the density $\rho^{[+]}$, for different values of $\rho^{[-]}$, is illustrated in figures $6 \mathrm{a}$ and $6 \mathrm{~b}$. In figure $6 \mathrm{a}$, we show simulation results for low values of $\rho^{[-]}$. Let us first describe the case where the heavy vehicles are absent. So, when $\rho^{[+]}$is below $\rho_{c}^{[+]}$, head-on collisions occur most unlikely. Above $\rho_{c}^{[+]}$, the PHC increases with increasing more the density, reaches a maximum, and then decreases until it vanishes when $\rho^{[+]}$exceeds some high density value. When heavy vehicles are present in the circuit, a peak appeared in the probability diagram, which is located in the very low density region. Indeed, when both $\rho^{[-]}$and $\rho^{[+]}$are low, the condition "Space2" occurs more likely if the heavy vehicles are absent and more unlikely when at least one heavy vehicle is present. With increasing more $\rho^{[+]}$, the PHC decreases and vanishes when $\rho^{[+]}$exceeds some high density value. At fixed relatively high density, the PHC increases with increasing the number of heavy vehicles present in the road. Clearly, the increase of the number of heavy vehicles in the circuit gives rise to the formation of additional jams behind heavy vehicles. This encourages cars $^{[+]}$to perform lane-changing in order to pass the heavy vehicles. At the same time, the occurrence of jams in the home lane will put at disadvantage the return of passing cars ${ }^{[+]}$. Therefore, at relatively high $\rho^{[+]}$, the increase of the proportion of heavy vehicles will increase the risk of head-on collision.

In figure $6 \mathrm{~b}$, we show simulation results for the PHC when $\rho^{[-]}$is relatively high. We find that the PHC exhibits a single peak around some low value of $\rho^{[+]}$. This means that head-on collision occurs most likely if $\rho^{[+]}$is very low. Furthermore, we observe that the peak is more pronounced if no heavy vehicle is present in the road. Indeed, at very low densities, the frequency of lane-changing of cars $^{[+]}$is more important in the case of the presence of heavy vehicles than in the case of their absence (Fig.3b). In these circumstances, the presence of only one heavy vehicle in the circuit can reduce significantly the risk of head-on collision. We find also that $\mathrm{PHC}$ is insensitive to the variation of the proportion of heavy vehicles. Indeed, when $\rho^{[-]}$is relatively high, most of head-on collisions are provoked by passing oncoming $\operatorname{cars}^{[-]}$ which faced the vehicles ${ }^{[+]}$on the home lane. When the home lane density 
becomes relatively high, no interaction will exist between lanes and then no head-on collision will occur.

\section{Conclusion}

In summary, we have investigated the risk of collisions between vehicles in two-lane bidirectional traffic flow models. We present conditions for the occurrence of three different collisions: rear-end collision, lane-changing collision and head-on collision. The density of vehicles on the two lanes and the proportion of heavy vehicles play an important role in the risk of car accident.

When the density on the oncoming lane is low, more available free space exist on the oncoming lane. If the home lane density is relatively high, the fast cars are intended to overcome the slow ones. This provokes the occurrence of dangerous situations in both lanes. So, in these situations, head-on collisions between passing cars and oncoming cars are very likely to occur. The unexpected return of cars to their home lane may cause lane-changing collisions. The rear-end collision may also occur because of the formation of additional jams on the home lane. It is found that, in bi-directional traffic, the probability of the rear-end collision is significantly higher than that of the head-on collision or the lane-changing collision. Nevertheless, the two last collisions are an often fatal type of road traffic accident whereas the rear-end collision may not result in catastrophic injury. Slowly moving heavy vehicles cause additional delay on the home lane and thus prevent the fluidity of traffic flow. This provokes a diminution of the cars speeds, and then promotes the pass of cars on the oncoming lane. Therefore, the risk of vehicle collisions should increase with increasing the proportion of heavy vehicles.

When the density of the oncoming lane is relatively high, oncoming cars can pass on the home lane whenever the density of this last is low. This can provoke collisions with vehicles travelling on the home lane. Here also, the presence of heavy vehicle can enhance the risk of car accidents; especially for the rear-end and the head-on collisions.

Because it is sometimes impossible to evaluate the risk of collisions using real crash data, the present study provides a simulation approach to investigate the probability of collision for bidirectional traffic accidents. 


\section{References}

[1] D. Chowdhury, L. Santen, A. Schadschneider, Physics Report 329, 199 (2000).

[2] S. Maerivoet and B.D. Moor, Phys. Rep. 4191 (2005).

[3] K. Nagel and M. Schreckenberg, J. Physique I, 2, 2221 (1992).

[4] D. Helbing, Rev. Mod . Phys., 731067 (2001).

[5] T. Nagatani, Rep. Prog. Phys. 65, 1331 (2002).

[6] P. Wagner, K. Nagel and D. E. Wolf, Physica A 234, 687 (1997).

[7] K. Nagel, D. E. Wolf, P. Wagner and P. Simon, Phys. Rev. E, 58, 1425 (1998).

[8] M. Rickert, K. Nagel, M. Schreckenberg and A. Latour, Physica A 235, 417 (1997).

[9] N. Moussa and A.K. Daoudia, Eur. Phys. J. B 31, 413 (2003).

[10] P.M. Simon and H.A. Gutowitz, Phys. Rev. E, 57, 2441 (1998).

[11] N. Moussa, Phys. Lett. A 372, 6701 (2008).

[12] N. Boccara, H. Fuks and Q. Zeng, J. Phys. A 30, 3329 (1997).

[13] D.W. Huang, J. Phys. A, 31, 6167 (1998).

[14] D. W. Huang and W. C. Tseng, Phys. Rev. E, 64, 057106 (2001).

[15] D-W. Huang and Y-P. Wu, Phys. Rev. E, 63, 022301 (2001).

[16] X-Q. Yang and Y-Q. Ma, Mod. Phys. Lett. B, 16, 333 (2002).

[17] R. Jiang, X-L Wang and Q-S Wu, J. Phys. A 36, 4763 (2003).

[18] X-q. Yang, Y-q. Ma, J. Phys. A 35, 1 (2002). 
[19] N. Moussa, Phys. Rev. E 68, 036127 (2003).

[20] R. Jiang, B. Jia, X-L Wang and Q-S Wu, J. Phys. A 37, 5777 (2004).

[21] N. Moussa, Int. J. Mod. Phys. C 16, 1133 (2005).

[22] X-Q Yang, Y-Q Ma and Y-M Zhao, J. Phys. A 37, 4743 (2004).

[23] W. Zhang, X-Q Yang, D-P Sun, K. Qiu and H. Xia, J. Phys. A 39, 9127 (2006).

[24] X-Q Yang, W. Zhang, K. Qiu, W-T Xu, G. Tang and L. Ren, Physica A 384, 589 (2007).

[25] X-Q Yang, Y-Q Ma, and Y-M Zhao, Phys. Rev. E 70, 046121 (2004).

[26] X-Q Yang, W. Zhang, K. Qiu, and Y-M Zhao, Phys. Rev. E 73, 016126 (2006). 


\section{Figures captions}

Fig.1. Illustration of the quantities relevant for the lane changing rules in the two-lane bidirectional traffic system.

Fig.2. Flow of vehicles ${ }^{[+]}$as a function of the density of vehicles ${ }^{[+]}$: (a) The density of cars $^{[-]}$is low enough $\left(\rho^{[-]}=0.05\right)$, (b) The density of cars $^{[-]}$is high enough $\left(\rho^{[-]}=0.30\right)$.

Fig.3. The dependence on the density of vehicles ${ }^{[+]}$of the lanechanging frequency of cars $^{[+]}$: (a) The density of cars $^{[-]}$is low enough $\left(\rho^{[-]}=0.05\right)$, (b) The density of cars ${ }^{[-]}$is high enough $\left(\rho^{[-]}=0.30\right)$.

Fig.4. The dependence on the density of vehicles ${ }^{[+]}$of the probability of rear-end collision: (a) The density of cars $^{[-]}$is low enough $\left(\rho^{[-]}=0.05\right)$, (b) The density of cars ${ }^{[-]}$is high enough $\left(\rho^{[-]}=0.30\right)$.

Fig.5. The dependence on the density of vehicles ${ }^{[+]}$of the probability of lane-changing collision: (a) The density of cars $^{[-]}$is low enough $\left(\rho^{[-]}=0.05\right)$, (b) The density of $\operatorname{cars}^{[-]}$is high enough $\left(\rho^{[-]}=0.30\right)$.

Fig.6. The dependence on the density of vehicles ${ }^{[+]}$of the probability of head-on collision: (a) The density of $\operatorname{cars}^{[-]}$is low enough $\left(\rho^{[-]}=0.05\right)$, (b) The density of cars $^{[-]}$is high enough $\left(\rho^{[-]}=0.30\right)$. 


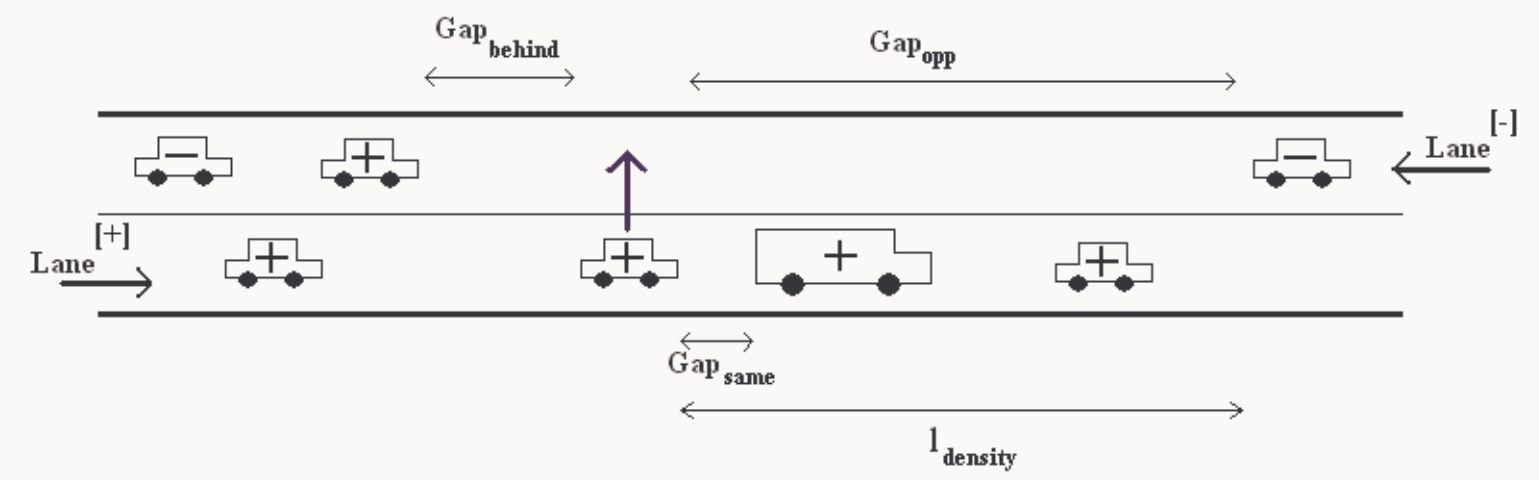

Figure 1 


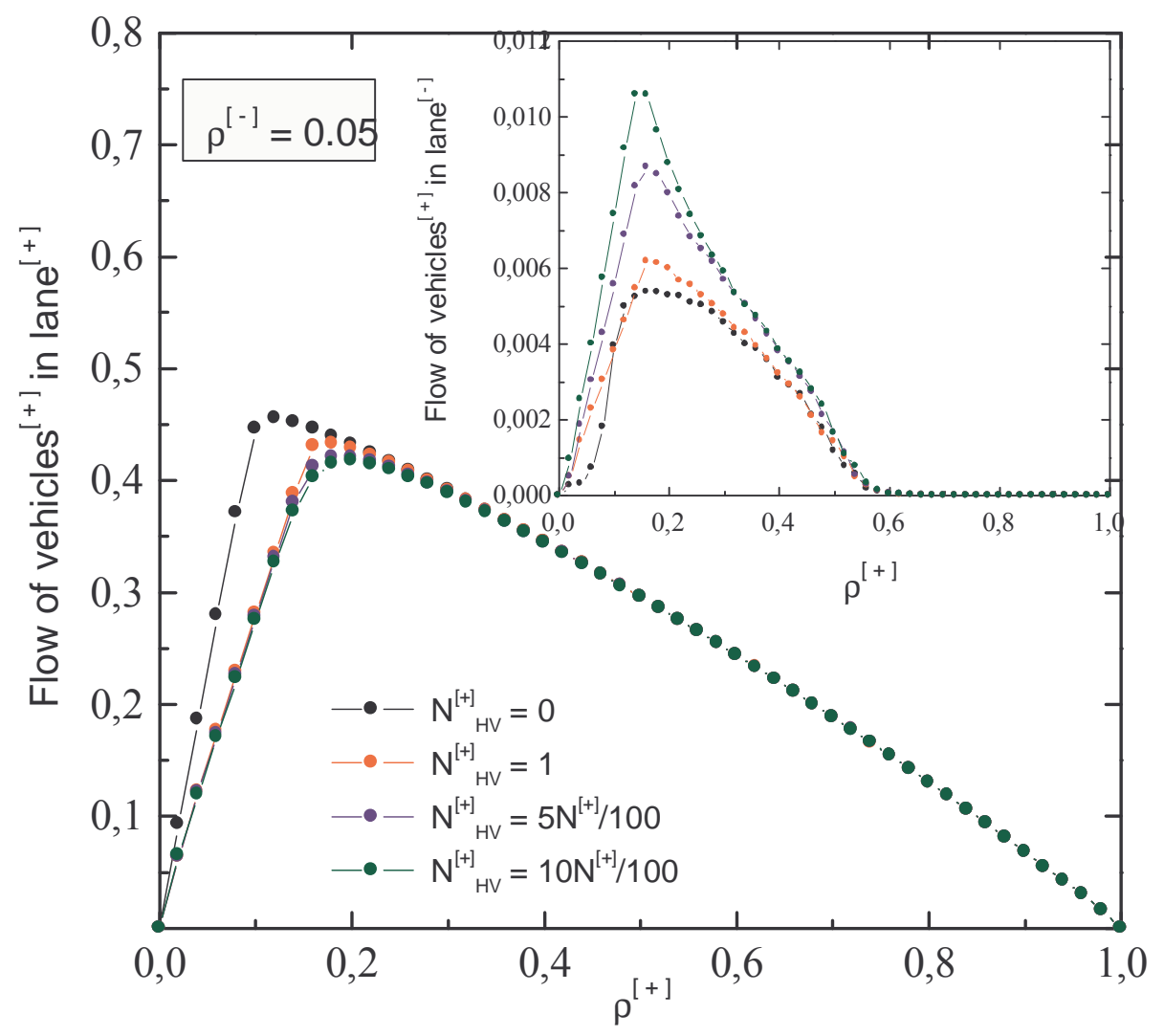

Figure 2a 


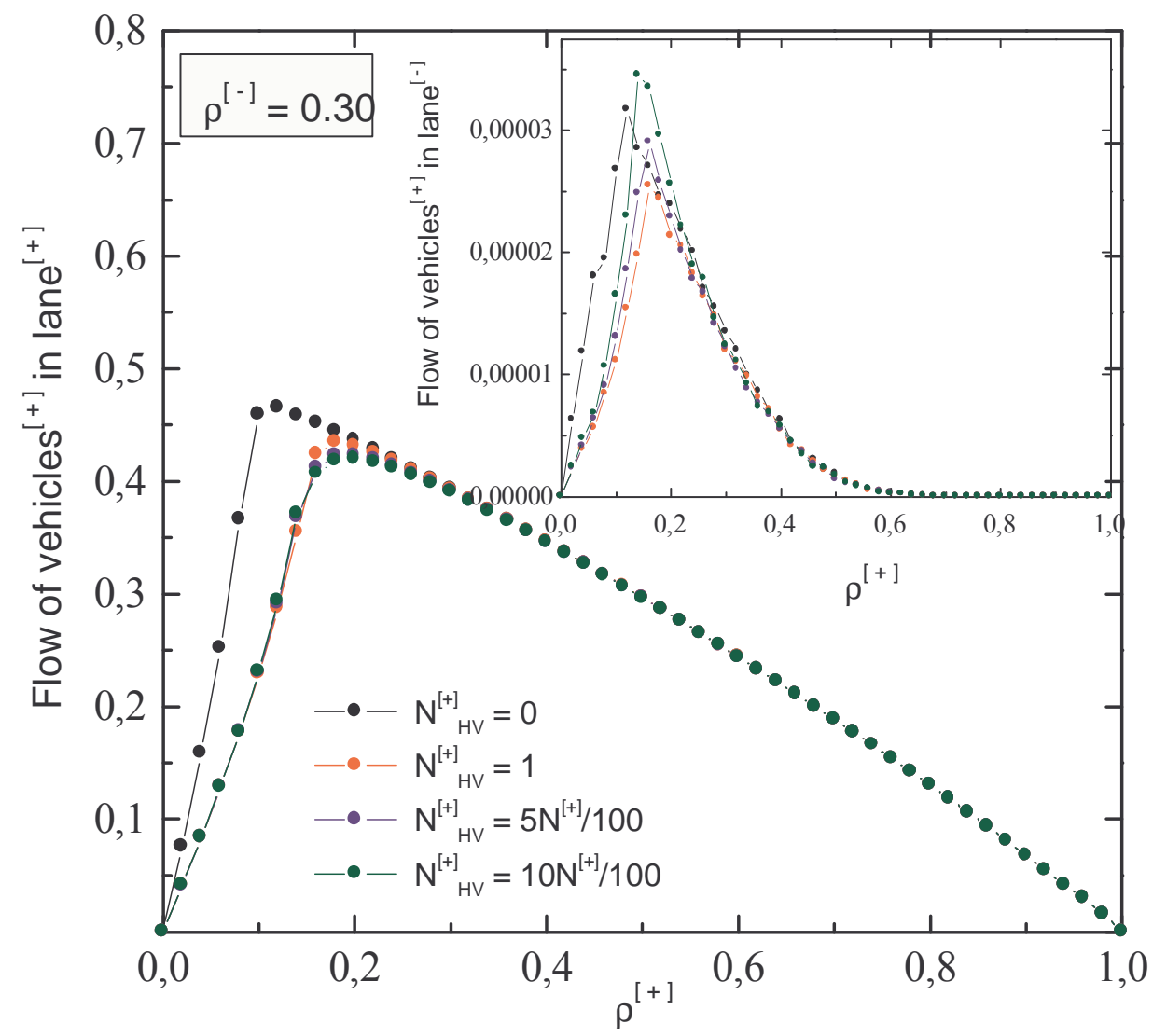

Figure $2 b$ 


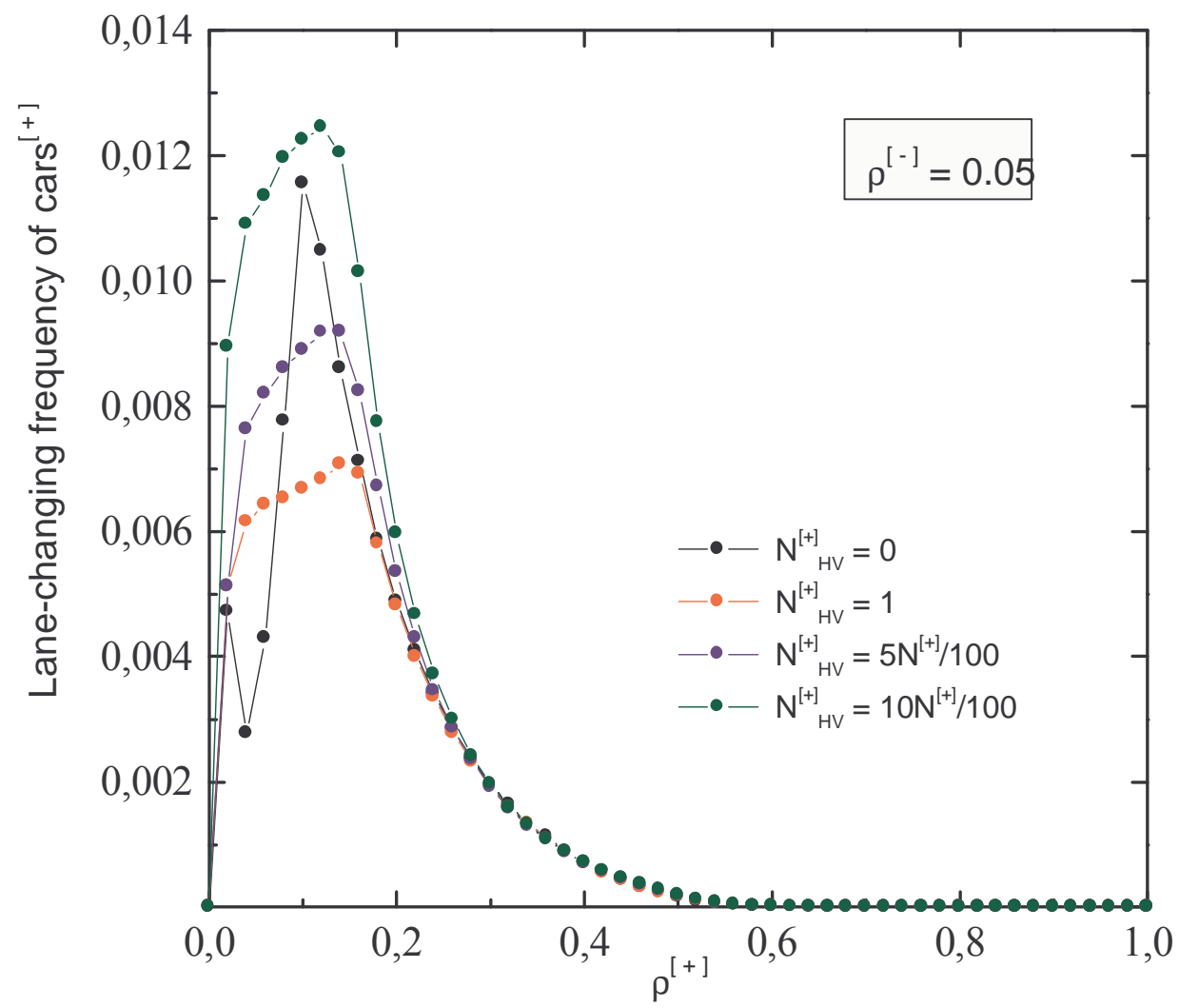

Figure $3 a$ 


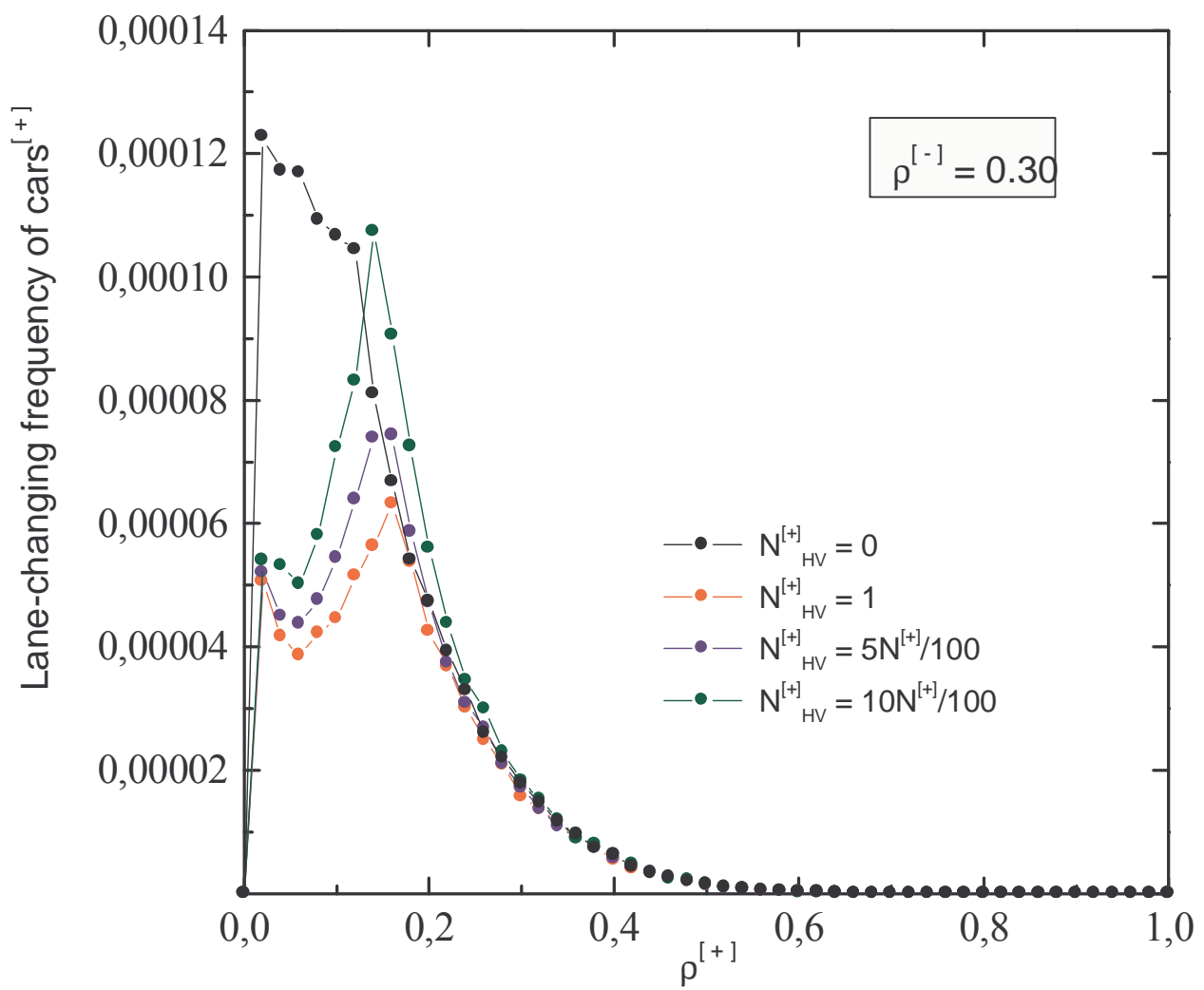

Figure $3 b$ 


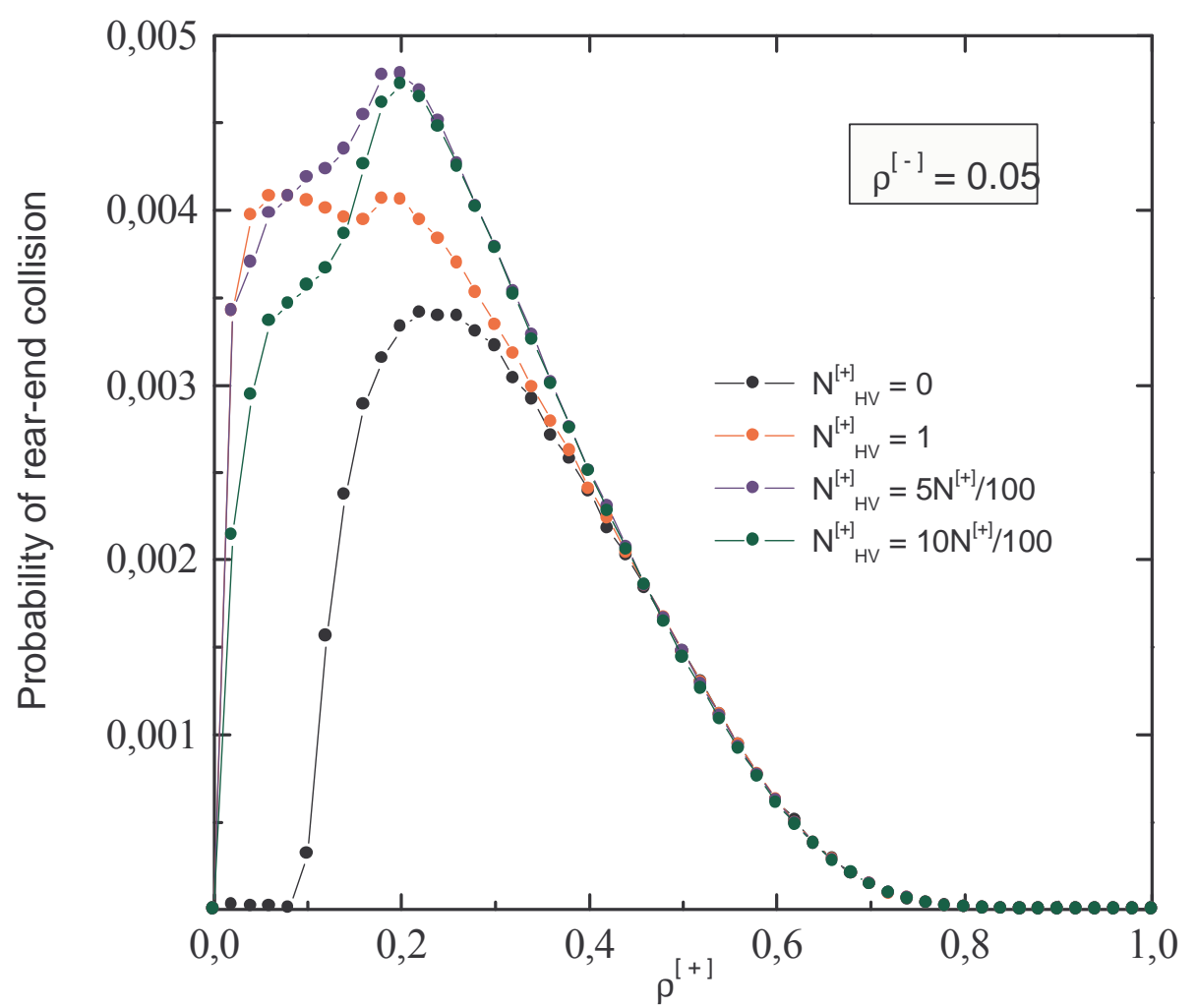

Figure 4a 


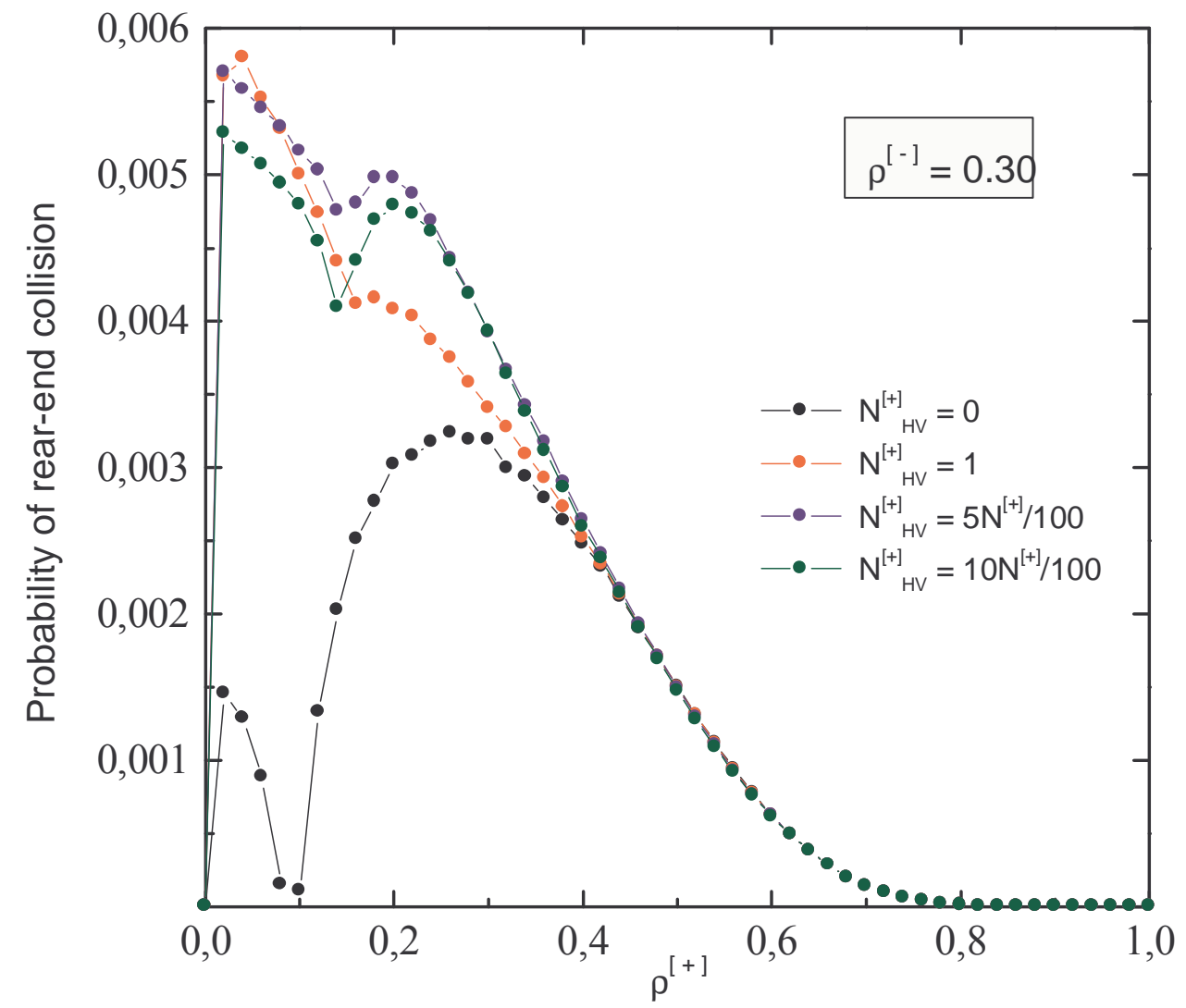

Figure 4b 


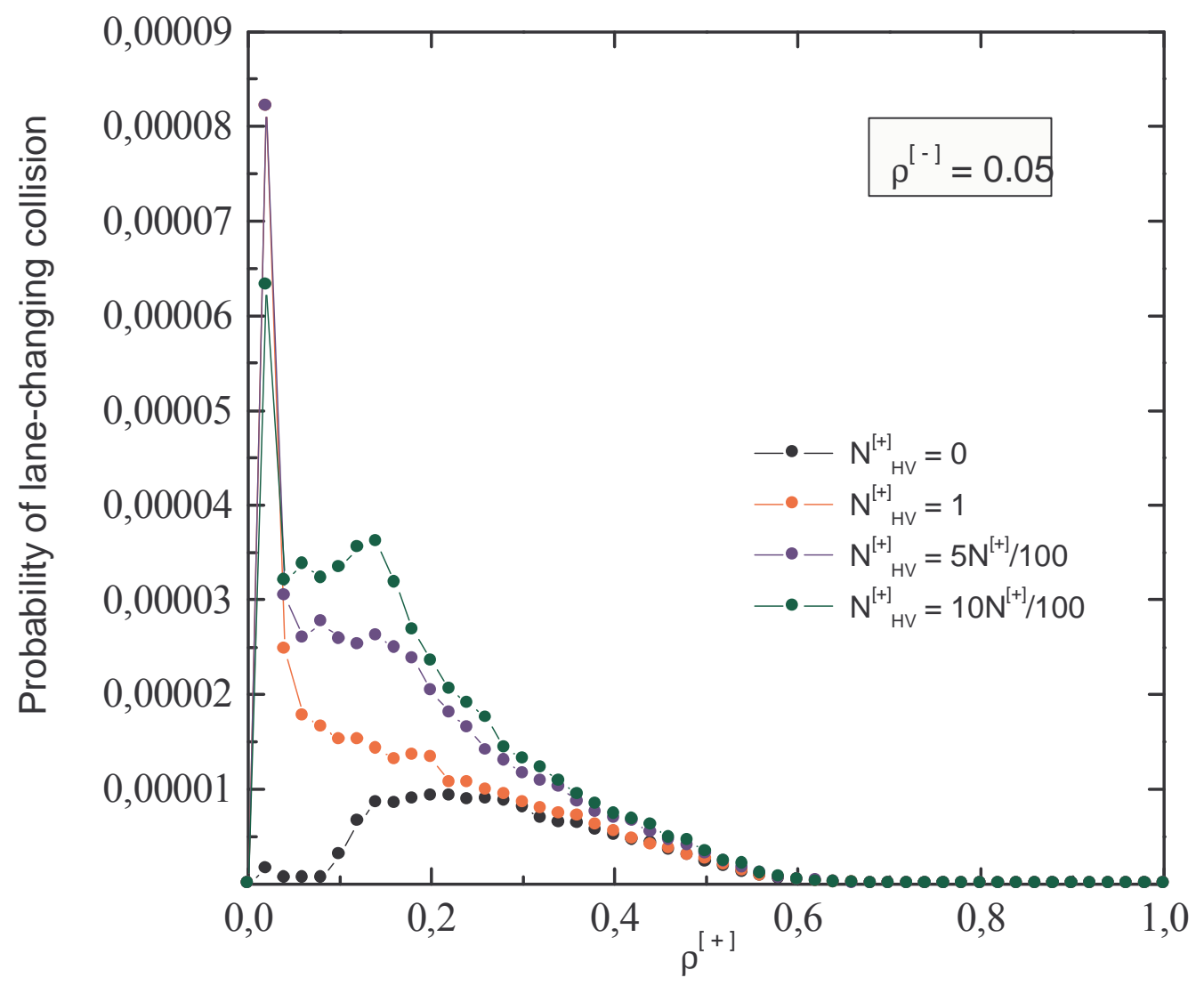

Figure 5a 


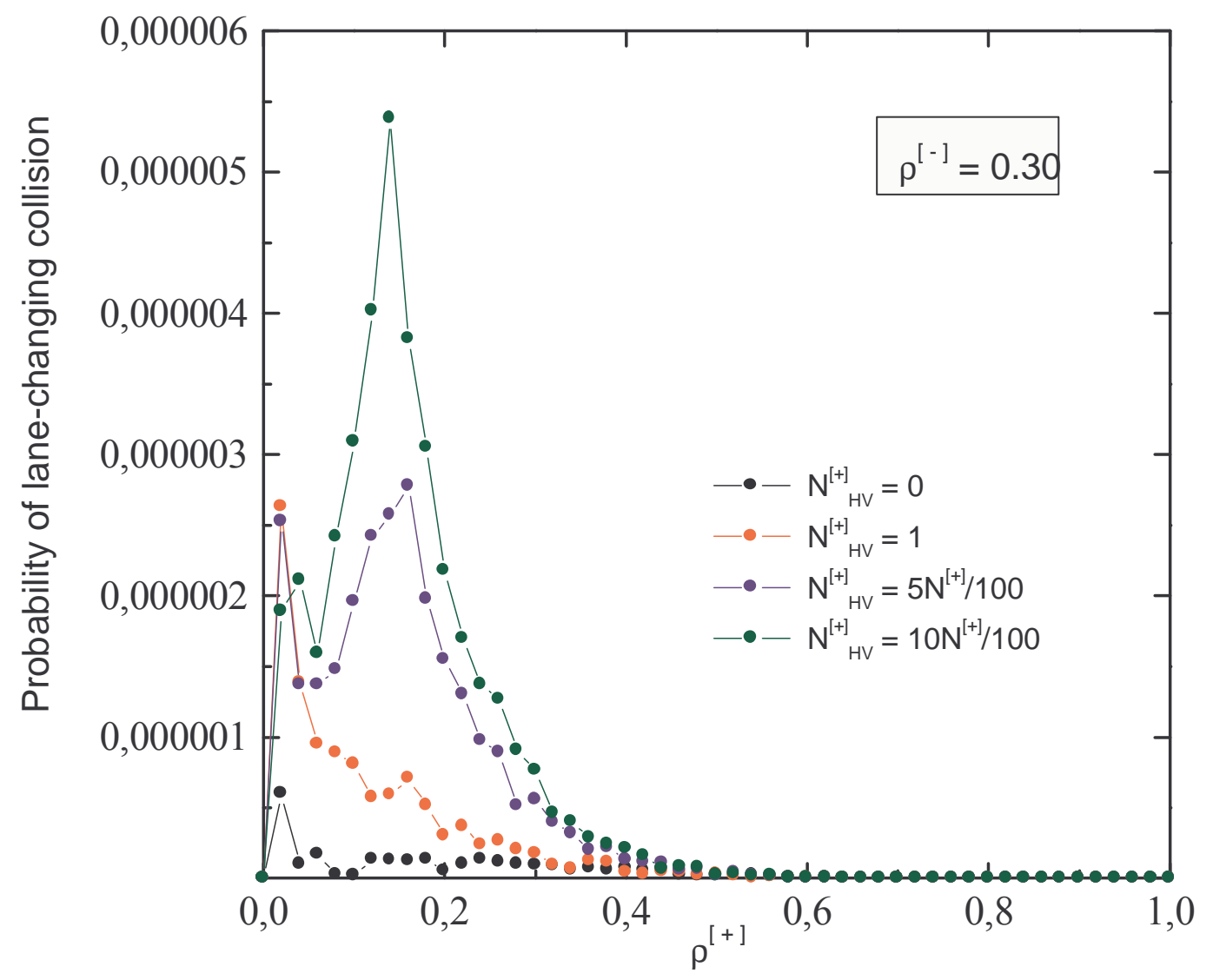

Figure 5b 


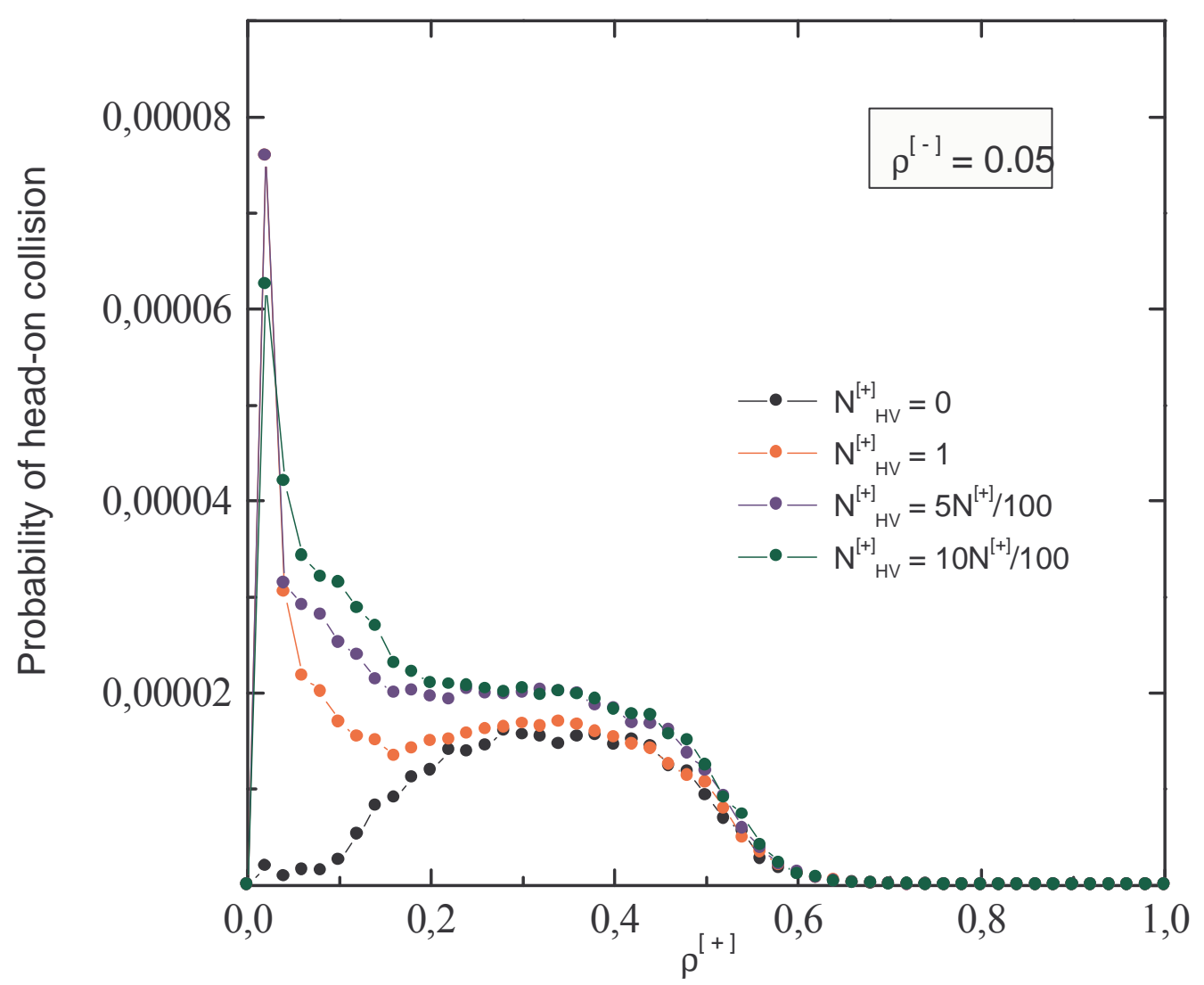

Figure $6 a$ 


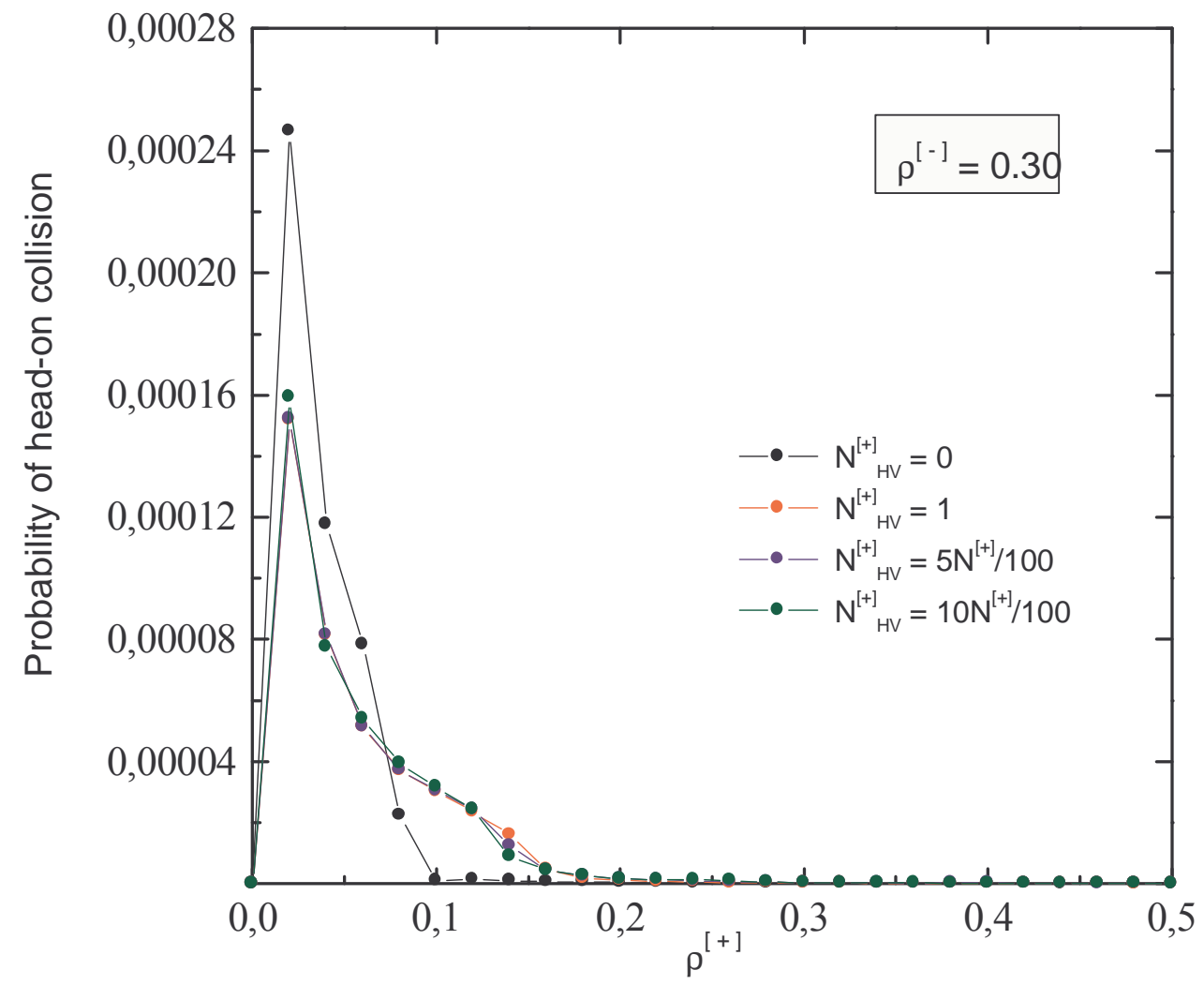

Figure 6b 